\title{
Verification Failures: What to Do When Things Go Wrong
}

\author{
Valeria Bertacco \\ University of Michigan \\ Department of Electrical Engineering and Computer Science \\ 2260 Hayward Avenue, Ann Arbor, MI \\ valeria@umich.edu
}

\begin{abstract}
Every integrated circuit is released with latent bugs. The damage and risk implied by an escaped bug ranges from almost imperceptible to potential tragedy; unfortunately it is impossible to discern within this range before a bug has been exposed and analyzed. While the past few decades have witnessed significant efforts to improve verification methodology for hardware systems, these efforts have been far outstripped by the massive complexity of modern digital designs, leading to product releases for which an always smaller fraction of system's states has been verified. The news of escaped bugs in large market designs and/or safety critical domains is alarming because of safety and cost implications (due to replacements, lawsuits, etc.).

This talk presents our solutions to solve the verification challenge, such that users of future designs can be assured that their devices can operate completely free of bugs. We attack the problem both at designtime, by presenting techniques to boost the fraction of the state space that can be verified before tape-out, and after deployment in the field, discussing novel solutions which can correct escaped bugs after a system has been shipped. Our ultimate vision for this technology is to make hardware as malleable as software.
\end{abstract}

